\title{
Starting with a Monte Carlo Analysis Disturbance on Multiple Rocket Spread
}

\author{
Keju Zhang ${ }^{1, a}$, Yunhua $\mathrm{Gao}^{2, \mathrm{~b}}$ \\ ${ }^{1}$ College of Information Technology, Shenyang Institute of Technology, Fushun 113122, China \\ ${ }^{2}$ Department of Mechanical and Electrical Engineering, Tangshan Vocational and Technical College, \\ Tangshan 064002, China \\ azhangkejuer@163.com, ${ }^{\mathrm{b}}$ gyhhot@gmail.com
}

Keywords: Initial disturbance, the Monte Carlo method, random sampling, landing error.

\begin{abstract}
Multi-tube rocket projectiles scattered in a considerable number of cases is mainly caused by the initial disturbance. Monte Carlo method is based on probability and mathematical statistics, based on probabilistic models do a mathematical simulation method to approximate calculation. It is a random sampling method as a means of reliability prediction system based on reliability block diagram. MATLAB simulation analysis using the deviation caused by the placement of the main error sources can save the development expenditure, but also shorten the development cycle.
\end{abstract}

\section{Introduction}

Initial disturbance is in the starting angle shot projectile point $\psi_{0}$, the starting angle of attack $\delta_{0}$, and its angular velocity $\dot{\delta}_{0}$. These quantities are all launching the projectile from the bore after the process began to move to the end of the period of limitation, a projectile by the action of various factors on the disturbance caused. Initial disturbance refers to the parameters related to the initial starting conditions outside the projectile trajectory equations corresponding to the six degrees of freedom as a projectile equation have 12 initial parameters, namely three rotation angle, three angular velocity, displacement and 3 lines 3 line speed. Therefore the initial disturbance, including starting the swing angle, initial swing angular velocity, initial angle, angular velocity and the initial disturbance and displacement, linear velocity, angular velocity from the angle and rotation.

\section{Monte Carlo Study Multiple Rocket Spread}

The main impact of the initial disturbance factors are: (1) missile and artillery gap; (2) projectile static and dynamic unbalance unbalanced; (3) the muzzle pressure $P_{g}$; (4) artillery; (5) projectile distance centering portion and an ammunition belt (or lower centering part) between; distance (6) The distance between projectile center and bomb belt (or lower centering portion) $l_{c}$; (7) for the horizontal axis of inertia by playing with a center (8) resistance arm; (9) projectile speed $r_{0}$.

In order to reduce the initial disturbance, should properly adjusting the various parameters in the overall design of artillery systems, especially some deviation factors such as missile and artillery exercise space, projectile, static unbalance, artillery vibration, etc., should be done properly controlled, using closed loop installed on gas bombs, mortar shells using low-speed rotation and other measures proved to reduce the spread of projectiles have better results. When the amount of the reliability characteristics of each unit is known, but the reliability of the model system is too complex and difficult to derive a general equation can be solved, the Monte Carlo method can be probabilistic unit to complete the task and reliability block diagram, calculate the approximate the reliability of the system. Because this method requires trial and error, working too cumbersome, so it is always done with a computer. 


\section{The computer simulation of Initial Disturbance on the Multiple Rocket}

An experiment using multiple rocket system will consume large mounts of bullets, so to minimize the number of tests without affecting the results of the case. In this case, in order to predict and assess whether the technical indicators hit precision, analytical bias caused by the placement of the main sources of error, the computer is essential for a digital simulation techniques can save research expenditure, but also shorten the development cycle.

Shooting Accuracy of Multiple Rocket. Shooting accuracy MLRS include shooting accuracy and shooting intensity. In the multi-barrel rocket launchers and flight dynamics calculation takes into account the influence of various factors on the random placement and intensity of rockets, and after multiple rocket launchers and flight dynamics stochastic simulation, we can get all rockets longitudinal and lateral placement coordinates are $\left(X_{1}, X_{2}, \ldots \ldots X_{n}\right)$ and $\left(Z_{1}, Z_{2}, \ldots \ldots Z_{n}\right)$, where $\mathrm{n}$ is the number of firing hair. Hutchison rockets were longitudinal, transverse and average placement position, shooting intensity for and. Then get the average intensity of the impact point and shoot rockets.

$$
\begin{aligned}
& \bar{X}=\frac{1}{n} \sum_{i=1}^{n} X_{i}, \bar{Z}=\frac{1}{n} \sum_{i=1}^{n} Z_{i} \\
& E_{X}=0.6745 \sqrt{\frac{\sum_{i=1}^{n}\left(X_{i}-\bar{X}\right)}{n-1}}, E_{Z}=0.6745 \sqrt{\frac{\sum_{i=1}^{n}\left(Z_{i}-\bar{Z}\right)^{2}}{n-1}}
\end{aligned}
$$

The inherent mechanical properties of a variety of random factors and multi-barrel rocket weapon system (including rockets loading position, speed, shooting interval, etc.) are the main factors affecting the intensity of the shooting. Motion disturbance factors rockets into two non-randomness randomness. The former can not be determined in advance, such as wind gusts, the initial disturbance, thrust eccentric, uneven mass distribution, (random changes caused by various hair between ignition systems) and other random shooting interval; latter is pre-determined, such as the average deviation of temperature, pressure, playing heavy bias, rockets loading position, shot sequencing.

The Simulation of Initial Disturbance The simulation model identifies the location coordinates of the projectile, and then on the circle marked the placement of the target projectile. The radius of the circle of the target design is the application of statistical methods to transmit five group projectile, its placement position statistics, these five points and the mean distance from the bull's-eye as the inner layer of the second ring (the target total by five rings) radius, and then draw a circle target. Meanwhile placement round target distance is given below. After the $n$ times point shot hit the statistics mean and variance. This paper, using the method of Monte Carlo sampling test to determine the statistical features of the projectile hit points (mean and variance). In the initial speed of $300 \mathrm{~m} / \mathrm{s}$, we simulated by changing the angle of trajectory and heading angle.

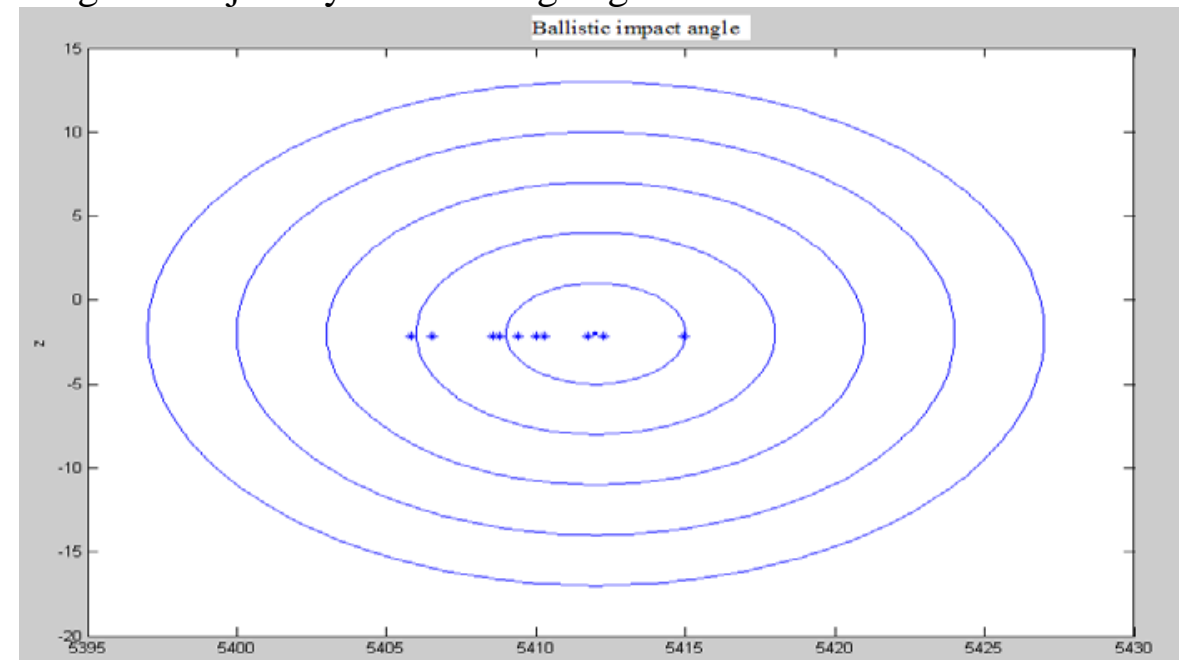

Fig. 1 Ballistic impact Angle 
(1) Initial disturbance trajectory angle.

In MATLAB, run the program which only consider the trajectory angle change, when to get in, Figure 1.

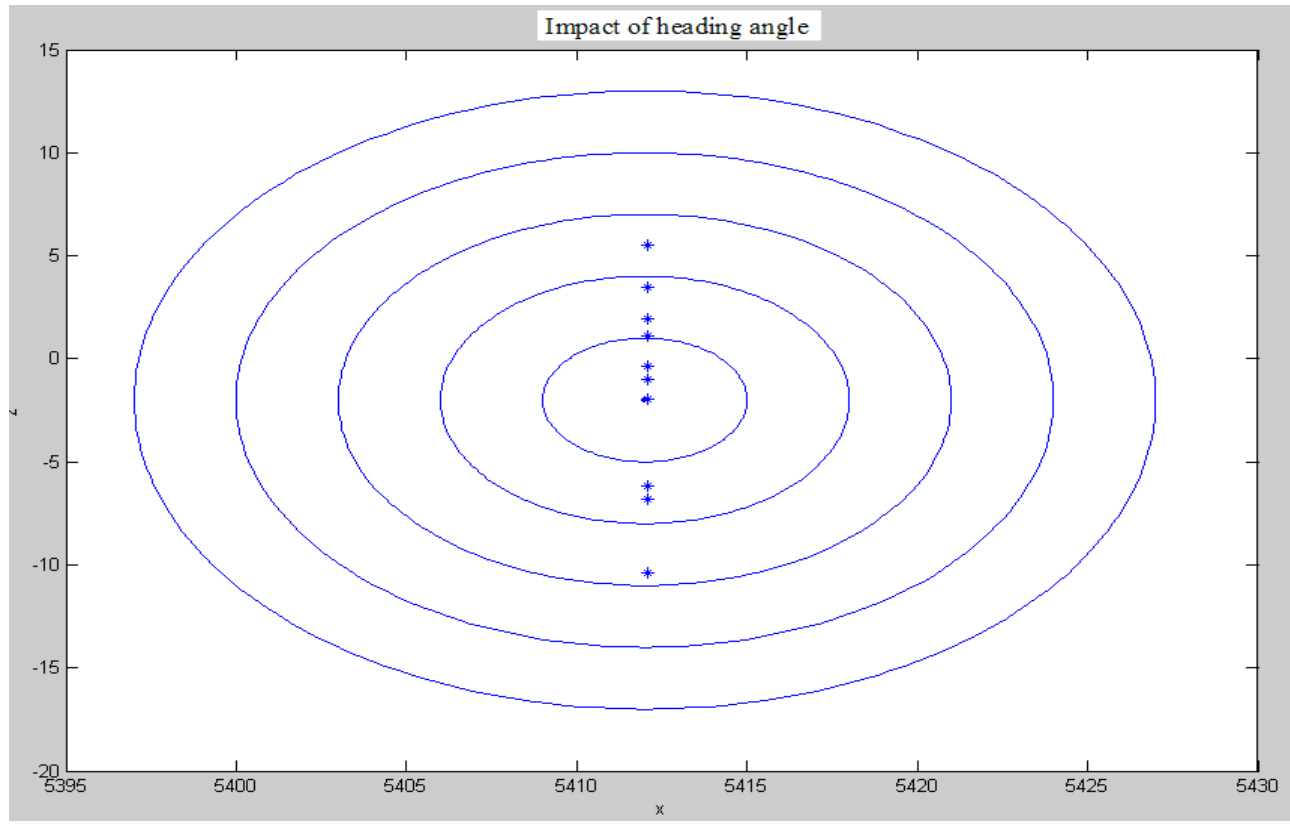

Fig. 2 Impact of heading Angle

(2) The initial disturbance heading angle

In MATLAB, to run the program in which only consider the change of the course angle. In Fig 2, $E_{X}=14.732, D_{X}=127.598$.

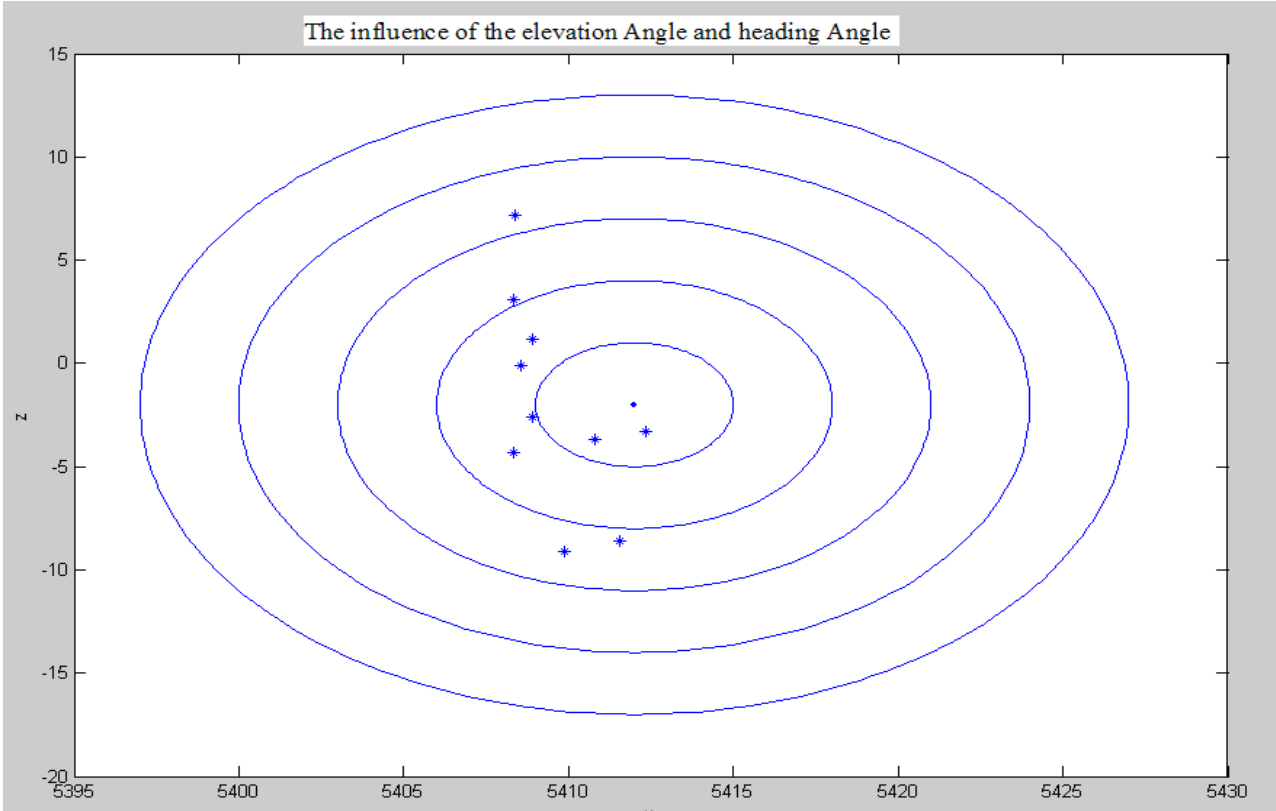

Fig. 3 The influence of the elevation Angle and heading Angle

(3) The initial disturbance trajectory and heading angle of inclination

In MATLAB, the simulation program considering initial disturbance elevation Angle and heading Angle is on Figure 3. 


\section{Results Analysis}

In this paper, the Monte Carlo method for sampling tests to determine the statistical properties of projectile hit points (mean and variance) in order to analyze the impact of the projectile initial disturbance intensity of the impact point. In the initial speed of $300 \mathrm{~m} / \mathrm{s}$, get a different trajectory simulation graphics by changing the angle of inclination and heading. Trajectory inclination and heading angle, the trajectory inclination greater impact on the placement of intensity, and heading angle of impact on the placement density is small. Therefore, to make the projectile hit the target more accurately, it should be major improvements trajectory inclination for placement density of the initial disturbance.

\section{References}

[1] X. F. Qian, R. X. Lin, Y. N. Zhao. Missile flight mechanics Beijing: Beijing Institute of Technology Press, 2002.

[2] Z. S. Shen, S. Zhong, Y. F. Liu. Projectile aerodynamics Nanjing: Nanjing University Press, .2005

[3] AA Dmitriyevich Ostrovsky, Л.H Ray Tymoshenko, CC Porco Gisborne Rostov. National Defense Industry Press, 2000. 